\title{
Las enseñanzas del acertijo de las doce monedas
}

\section{(2a. parte)}

\author{
M.A. Murray-Lasso \\ Unidad de Enseñanza Auxiliada por Computadora \\ Departamento de Ingeniería de Sistemas. División de Estudios de Posgrado \\ Facultad de Ingeniería, UNAM
}

(recibido: abril de 2001; aceptado: septiembre de 2001)

\begin{abstract}
Resumen
Se presentan varios problemas prácticos relacionados con el acertijo de las doce monedas, entre los que se encuentran el problema del diagnóstico médico y los árboles óptimos de diagnóstico. Dicho problema es importante en teoría de sistemas y tiene aplicaciones al diseño de códigos óptimos de comunicación, diseño de algoritmos de intercalación (merge) y diseño de estructuras de datos óptimas para búsquedas en bases de datos. Se explica el papel que juegan conceptos centrales como árboles diagnósticos para estructurar la búsqueda de la solución, eliminación ordenada de conjuntos de posibilidades, información proporcionada por un experimento, conteo y balanceo de puntas del árbol, conversión del problema de diagnóstico en un problema de rutas más cortas en una red de estados y algunos algoritmos óptimos para estos problemas como el de rutas más cortas de Floyd y el algoritmo de códigos óptimos de Huffman.
\end{abstract}

Descriptores: acertijos matemáticos, diagnóstico, árbol de búsqueda, búsqueda ternaria, espacios de estado, ruta más corta.

\begin{abstract}
Several practical problems related to the twelve-coin puzzle are presented, among them, medical diagnosis and optimal diagnosis trees. The diagnosis problem is an important problem of Systems Theory and has applications in the design of optimal communication codes, design of merge algorithms and the design of optimal information structures for searching data bases. The roles played by centrral concepts such as diagnostic trees to structure the search for a solution, ordered elimination of sets of possibilities, information provided by an experiment, counting and balancing of tree ends, and conversion of the diagnostic problem into a shortest route problem in a network of states are explained and some optimal algorithms for these problems, such as the shortest route algorithm of Floyd and the optimal coding algorithm of Huffman are given.
\end{abstract}

Keywords: mathematical puzzles, diagnostics, search tree, ternary search, state space, shortest route.

\section{Introducción}

La importancia de los acertijos matemáticos comovehículo educativo, es que se pueden aprovechar las ideas y técnicas utilizadas para resolverlos y atacar otros problemas que guardan alguna similitud con el acertijo. Cada una de las pesadas se puede interpretar como una medición 
de síntomas que sirve para decidir si un defecto en particular puede ser descartado o no. Por ejemplo, en el problema de las doce monedas ( la. parte de este artículo), en el primer experimento en donde se pesaron las monedas 1 a 4 contra 5 a 8 , si la balanza se equilibraba podíamos concluir que ninguna de las primeras 8 monedas era defectuosa, en otras palabras, se descartaron las posibilidades de defecto siguientes: $1 \mathrm{~L}, 1 \mathrm{P}, 2 \mathrm{~L}$, $2 \mathrm{P}, 3 \mathrm{~L}, 3 \mathrm{P}, 4 \mathrm{~L}, 4 \mathrm{P}, 5 \mathrm{~L}, 5 \mathrm{P}, 6 \mathrm{~L}, 6 \mathrm{P}, 7 \mathrm{~L}, 7 \mathrm{P}, 8 \mathrm{~L}, 8 \mathrm{P}$, quedando solamente como posibles los defectos: $9 \mathrm{~L}, 9 \mathrm{P}, 10 \mathrm{~L}, 10 \mathrm{P}, 11 \mathrm{~L}, 11 \mathrm{P}, 12 \mathrm{~L}, 12 \mathrm{P}$. Podemos decir que el problema de las 13 monedas es una búsqueda tricótoma (por ser tres las ramas que generan cada pesada) que en cada experimento bien diseñado se eliminan $2 / 3$ de las posibilidades restantes y se reduce la búsqueda a $1 / 3$ de las posibilidades restantes. En el caso de la búsqueda binaria para localizar una llave en un archivo, cada experimento descarta la mitad (aproximadamente) de los registros restantes, y deja como posibles la otra mitad

El proceso iguala la labor de un médico al estar diagnosticando a un paciente. Si un determinado padecimiento se presenta junto con ciertas condiciones tales como alta temperatura, pulso acelerado y manchas en la piel, entonces el médico observando dichas condiciones puede descartar algunos padecimientos y reducir las enfermedades posibles.

\section{El diagnóstico médico, un problema análogo al acertijo de las monedas}

Una de las labores más importantes de un médico es diagnosticar a un paciente. Al conocer el padecimiento, el tratamiento y su supervisión se puede considerar como una labor de rutina. El desarrollo acelerado de la tecnología y su posible aplicación a la medicina, ha motivado una revisión cuidadosa de la práctica médica (Ledley y Lusted, 1959), lo cual ha desembocado en técnicas como los expertos artificiales (Shortliffe, 1976); (Levine et al., 1986) y las redes neuronales (Hopfield, 1982); (Allman, 1989) en las cuales los investigadores interesados en problemas médicos fueron pioneros
Desde el punto de vista abstracto, la situación del diagnóstico médico la podemos representar por medio de una tabla de síntomas y padecimientos como se muestra a continuación (Murray-Lasso, 1972); (De Vecchi, 1972):

Tabla de síntomas y padecimientos

\begin{tabular}{ccccc}
\hline \hline Síntomas $\backslash$ Padecimientos & P1 & P2 & P3 & P4 \\
\hline S1 & 0 & 0 & 1 & 1 \\
S2 & 1 & 0 & 0 & 1 \\
S3 & 0 & 1 & 1 & 1 \\
\hline \hline
\end{tabular}

En la tabla hemos llamado a los padecimientos P1, P2, P3 y P4 y a los síntomas S1, S2, S3. Los l's y 0 's indican la presencia o ausencia de los síntomas con el padecimiento correspondiente. Así, con el padecimiento Pl se presenta el síntoma S2 y no se presentan los síntomas SI y S3. Con el padecimiento P4, se presentan los tres síntomas S1, S2, y S3. El modelo es una aproximación a lo que sucede en la práctica. Un modelo más realista consideraría que los síntomas se presentan en una población determinada con una cierta frecuencia relativa; la aproximación consiste en considerar que si la frecuencia relativa es mayor que $50 \%$ se toma como que se presenta el padecimiento con el síntoma, y si es menor que $50 \%$, se toma como que no se presenta el síntoma con el padecimiento. Un modelo más realista puede tomar tres en lugar de dos regiones para las frecuencias relativas. Si la frecuencia relativa está entre 0 y $25 \%$, entonces se toma como que el síntoma no se presenta. Si está entre 75 y $100 \%$ el síntoma se presenta y si está entre 25 y $75 \%$ se toma como que "no importa" si el síntoma se presenta o no. Por facilidad usaremos el primer modelo.

Otra idea que apareció en la solución del acertijo de las monedas y que es útil en el diagnóstico médico, es el uso de los árboles para organizar los resultados y la idea de llevar cuenta de qué defectos siguen bajo consideración en las diferentes partes del árbol. Un posible árbol de diagnóstico que surge de la tabla es el siguiente: 


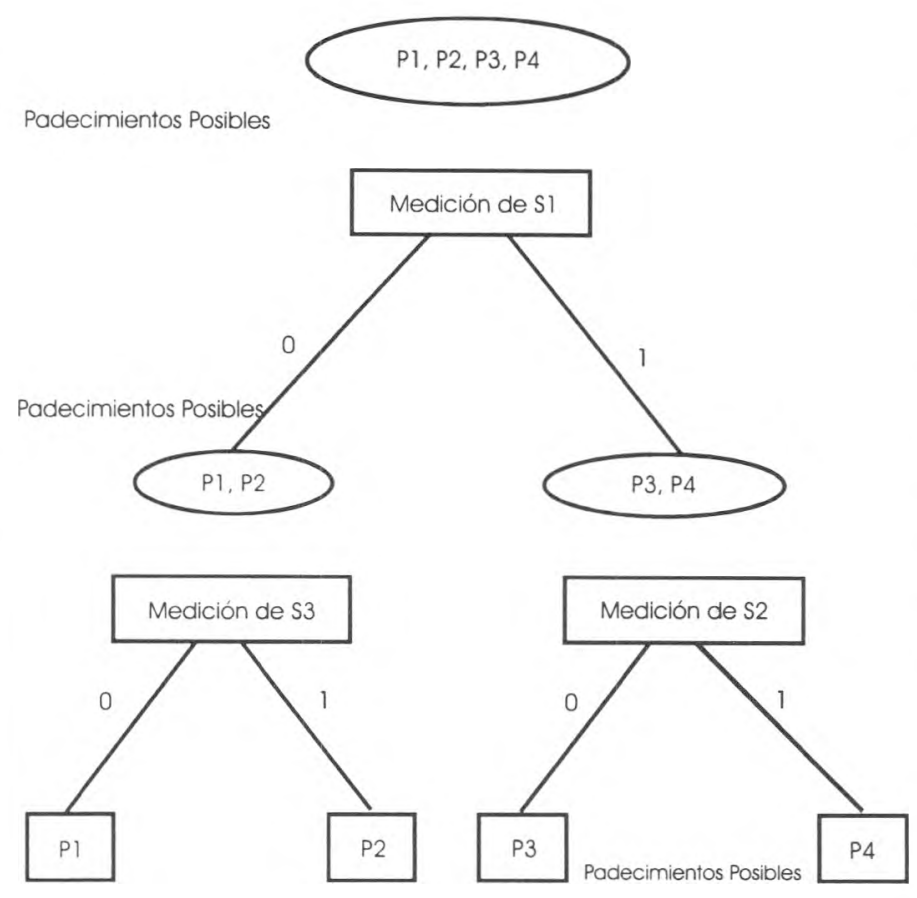

Figura 1

En la figura 1 después de dos mediciones tenemos una punta final para cada padecimiento, igual que teníamos un defecto después de 3 experimentos en el caso de las 12 monedas. En vez de que el árbol se trifurque en cada experimento (medición de síntoma) el árbol de la figura 1 solo se bifurca, debido a que las únicas posibilidades son: existe o no el síntoma. En el problema de las monedas no contábamos con una tabla de síntomas explícita (aunque, en principio la podríamos haber hecho, lo cual hubiera sido sumamente laboriosa por extensa). A cada posible resultado en un experimento, en la balanza le correspondería un síntoma. Por ejemplo, supóngase que se pesan la moneda 1 contra la 2 y que llamamos Síntoma 1 al resultado de que baje el plato en el que está la moneda 1. La relación con los 24 defectos (padecimientos) del acertijo de las monedas sería la siguiente:
El renglón que se exhibe, indica que se pueden eliminar 22 de los posibles defectos. (Puede sorprender al lector que siendo tan efectivo este experimento no se haya utilizado, sin embargo estamos analizando solamente una rama del árbol, la cual será tomada solamente en el caso que la moneda 1 o la 2 sea la defectuosa, situación poco probable; lo más probable es que la balanza quede en equilibrio, en cuyo caso solamente se eliminan 4 posibles defectos, quedando 20 vivos y gastando una de las pesadas). Además de tener la certeza de que las monedas 3 a 12 son buenas, podemos eliminar las alternativas $1 \mathrm{~L}$ y $2 \mathrm{P}$, pues si cualquiera de ellas fuera cierta, el experimento realizado no podría haber arrojado el resultado descrito (baja moneda 1 y sube moneda 2). Las posibilidades que quedan vivas con la presencia del síntoma son I Py $2 \mathrm{~L}$. Desgraciadamente el número de posibles síntomas es muy grande, solamente las posibilidades pesando una moneda contra otra son $(12 \times 11 \times 3) / 2=198$. Luego habría que considerar las posibilidades pesando dos monedas contra dos, luego tres contra tres, etc. De cada una de estas familias de experimentos existen muchas variantes, por ejemplo, si se pesan 6 monedas contra 6, hay que contar las distintas combinaciones de monedas que se pueden tener en cada plato de la balanza.

La solución del acertijo requiere que escojamos de entre todos esos posibles síntomas la combinación adecuada, para que cada camino del principio de un árbol a las puntas deje un solo defecto posible, similar al de la figura 1, en donde todos los defectos queden cubiertos y que el árbol no tenga más de 3 etapas (número de pesadas permitidas)

De una tabla de síntomas-padecimientos se pueden construir muchos árboles de diagnóstico parecidos al de la figura 1 ; en la figura 2 se muestra otro.

\begin{tabular}{||c|c|c|c|c|c|c|c|c|c|c|c|c|c|c|c|c|c|c|c|c|c|c|c|c|}
\hline \hline $\mathrm{Pi}$ & $1 \mathrm{~L}$ & $1 \mathrm{P}$ & $2 \mathrm{~L}$ & $2 \mathrm{P}$ & $3 \mathrm{~L}$ & $3 \mathrm{P}$ & $4 \mathrm{~L}$ & $4 \mathrm{P}$ & $5 \mathrm{~L}$ & $5 \mathrm{P}$ & $6 \mathrm{~L}$ & $6 \mathrm{P}$ & $7 \mathrm{~L}$ & $7 \mathrm{P}$ & $8 \mathrm{~L}$ & $8 \mathrm{P}$ & $9 \mathrm{~L}$ & $9 \mathrm{P}$ & $10 \mathrm{~L}$ & $10 \mathrm{P}$ & $11 \mathrm{~L}$ & $11 \mathrm{P}$ & $12 \mathrm{~L}$ & $12 \mathrm{P}$ \\
\hline $\mathrm{S} 1$ & 0 & 1 & 1 & 0 & 0 & 0 & 0 & 0 & 0 & 0 & 0 & 0 & 0 & 0 & 0 & 0 & 0 & 0 & 0 & 0 & 0 & 0 & 0 & 0 \\
\hline
\end{tabular}




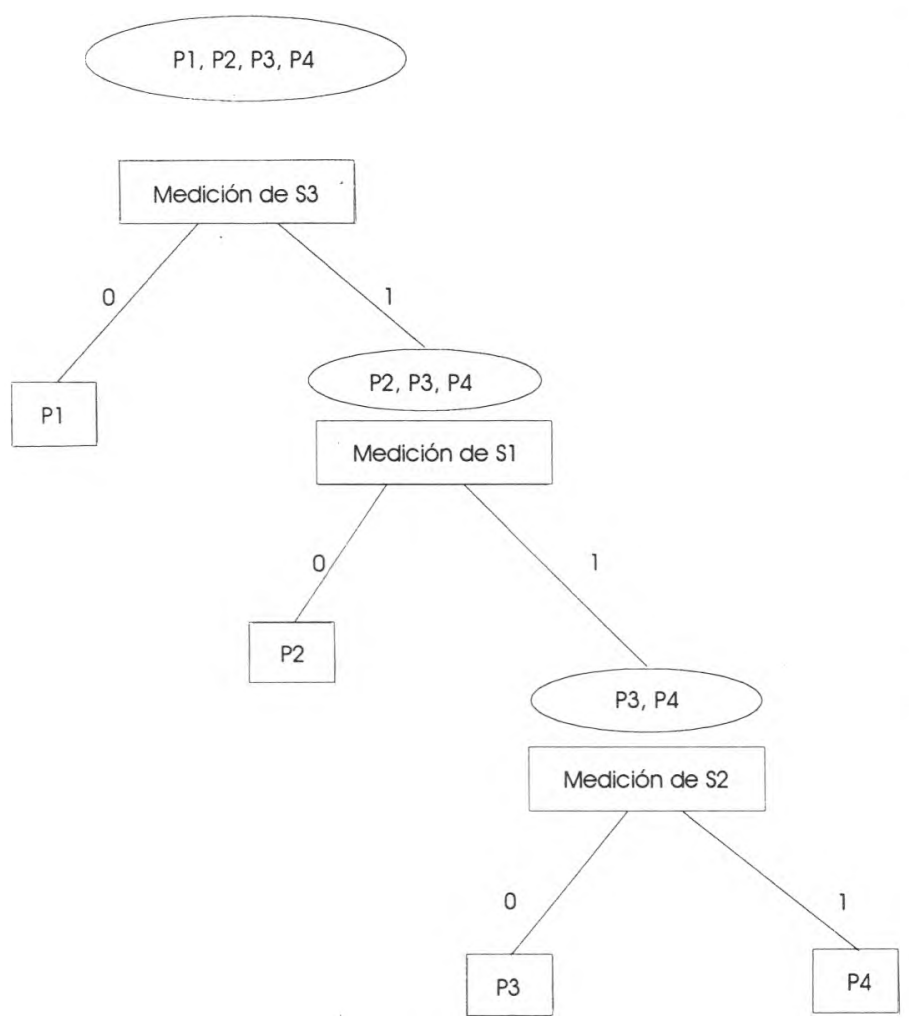

Figura 2

De los dos árboles de las figuras 1 y 2, ¿cuál es mejor? Todo depende del criterio de bondad. Si se exige que el diagnóstico se haga en dos mediciones o menos, el de la figura 2 quedaría descalificado. Si por otra parte, el padecimiento Pl fuera muy frecuente (por ejemplo gripe) mientras que los padecimientos P2, P3, y P4 fueran muy raros, entonces el árbol de la figura 2 sería mejor, pues en la inmensa mayoría de los diagnósticos éste se lograría midiendo únicamente un síntoma. También habría que tomar en consideración el "costo" de medir los síntomas. Por ejemplo, el síntoma S3 podría ser muy costoso. Aquí no solamente se puede considerar el costo en metálico de medir un síntoma (digamos, tomar la temperatura contra hacer un estudio de tomografía axial computada), sino también el costo en dolor o molestias para el paciente (hacerle una punción en la próstata contra un análisis con una muestra de sangre). Si medir S3 fuese muy costoso, se podría utilizar un árbol diagnóstico que solamente utilice los síntomas S1 y S2. (El árbol sería idéntico al de la figura 1 sustituyendo la medición de S3 por una medición de S2 e intercambiando el 0 y 1 en las ramas subsecuentes). Un buen modelo toma en consideración, tanto la frecuencia de los padecimientos como el costo de la medición de los síntomas.

\section{Conversión del diseño óptimo de árboles de diagnóstico en uno de rutas más cortas}

Un problema que tiene muchas aplicaciones y que puede ayudar en el diseño de árboles de diagnóstico, es el encontrar la ruta más corta entre un par de puntos en una red de carreteras. Para resolver este problema existen algoritmos eficientes y fáciles de programar.

Aunque por falta de espacio no entraremos en detalles en este artículo, en la figura 3 se muestra una red marcada con las distancias en tramos, para la cual se requiere encontrar la ruta más corta entre los puntos 7 y 1 . La red tiene 3 carreteras de un solo sentido marcadas con flechas. Las demás son de dos sentidos.

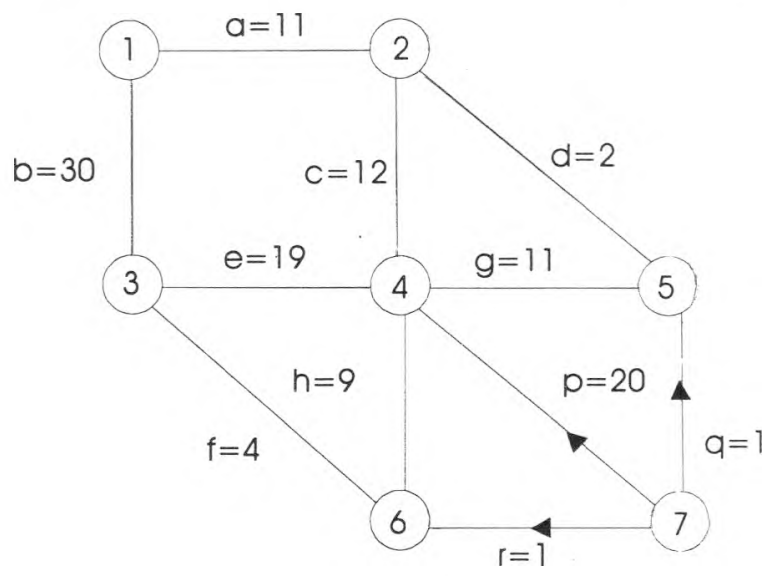

Figura 3

La siguiente matriz (Hu, 1969) fue obtenida usando un algoritmo para calcular todas las rutas más cortas de una red, dado por Floyd (1962) y muestra las longitudes de las rutas más cortas entre todos los pares de puntos. La componente $(i, j)$ nos da la longitud de la ruta que va del punto $i$ al punto $j$. Los números 9999 (que representan cantidades infinitas) indican que no se puede llegar a esos puntos debido a los sentidos contrarios.

$\begin{array}{lllllll}0 & 11 & 30 & 23 & 13 & 32 & 9999\end{array}$




$\begin{array}{rrrrrrr}11 & 0 & 25 & 12 & 2 & 21 & 9999 \\ 30 & 25 & 0 & 13 & 24 & 4 & 9999 \\ 23 & 12 & 13 & 0 & 11 & 9 & 9999 \\ 13 & 2 & 24 & 11 & 0 & 20 & 9999 \\ 32 & 21 & 4 & 9 & 20 & 0 & 9999 \\ 14 & 3 & 5 & 10 & 1 & 1 & 0\end{array}$

La siguiente matriz sirve para deducir las trayectorias de las rutas más cortas. La componente $(i, j)$ indica el primer nodo $x$ en la ruta más corta del nodo $i$ al nodo $j$; para averiguar el resto se consulta el primer nodo en la ruta de $x$ a $j$ (destino) y se repite la operación hasta llegar al destino. Así, para encontrar los nodos en la ruta más corta de 7 a 1 , se van encontrando los nodos en las posiciones $(7,1)=5,(5,1)=2,(2,1)=1$. Por lo tanto, la ruta está dada por los nodos $7,5,2,1$. Observando la figura 3 se comprueba que la longitud de la ruta es $1+2+11=14$, cantidad que coincide con el elemento $(7,1)$ de la matriz de distancias más cortas.

$\begin{array}{lllllll}1 & 2 & 3 & 2 & 2 & 2 & 7 \\ 1 & 2 & 4 & 4 & 5 & 4 & 7 \\ 1 & 6 & 3 & 6 & 6 & 6 & 7 \\ 2 & 2 & 6 & 4 & 5 & 6 & 7 \\ 2 & 2 & 4 & 4 & 5 & 4 & 7 \\ 4 & 4 & 3 & 4 & 4 & 6 & 7 \\ 5 & 5 & 6 & 6 & 5 & 6 & 7\end{array}$

¿Qué tiene que ver el problema de las rutas más cortas con el diagnóstico médico?

En vez de ciudades y carreteras, podemos crear un espacio de estados, donde el estado inicial corresponde a un conjunto con un solo miembro, conteniendo éste todos los padecimientos. El estado final es un conjunto con un número de miembros igual al número de padecimientos, un miembro para cada uno de los padecimientos. En símbolos, el estado inicial es $\{\{\mathrm{P} 1, \mathrm{P} 2, \mathrm{P} 3, \mathrm{P} 4\}\}$ y el estado final es $\{\{\mathrm{P} 1\},\{\mathrm{P} 2\},\{\mathrm{P} 3\},\{\mathrm{P} 4\}\}$. Los estados intermedios pueden ser conjuntos de subconjuntos de las posibles particiones en grupos de padecimientos. Por ejemplo, $\{\{P 1, P 2\},\{P 3$, $P 4\}\},\{\{P 1\},\{P 2, P 3, P 4\}\},\{\{P 1\},\{P 2\},\{P 3, P 4\}\}$.
A cada uno de estos conjuntos de padecimientos "no totalmente separados" se le puede "aplicar" la medición de un síntoma que separe algunos de los elementos de uno de sus subconjuntos. Así, en el árbol de la figura 3 se aplica la medición del síntoma S1 al subconjunto $\{\mathrm{P} 2, \mathrm{P} 3, \mathrm{P} 4\}$, el cual por el efecto de la medición separa dicho subconjunto en los dos subconjuntos $\{\{\mathrm{P} 2\} .\{\mathrm{P} 3, \mathrm{P} 4\}\}$. La red se va construyendo (dentro de la computadora) aplicándole al estado inicial todas las posibles mediciones de síntomas que "despedacen" algún subconjunto de ese estado, generando para cada caso nuevos estados, al mismo tiempo que se va aplicando un algoritmo de rutas más cortas, (por ejemplo el de Dijkstra (1959) al cual se le acelera con una heurística, de manera que cuando se llegue al estado final se tenga también la información de cuál es la ruta más corta del estado inicial al estado final. La "distancia de cada tramo de carretera" es una función de las frecuencias relativas de los padecimientos que todavía hay que separar, así como de los costos de medir los síntomas que se vayan usando para lograr la separación. (Murray-Lasso y Lara, 1975); (Lara-Tejeda, 1977).

Para la tabla de síntomas y padecimientos mostrada anteriormente, la red que se genera es la que se muestra en la figura 4. En dicha red, el nodo inicial $\left(\mathrm{P}_{1} \mathrm{P}_{2} \mathrm{P}_{3} \mathrm{P}_{4}\right)$ genera los nodos en el siguiente nivel hacia abajo, al aplicarle las mediciones de los síntomas que se muestran junto a las flechas que parten del nodo inicial. De los nodos recién generados surgen otros en el siguiente nivel hacia abajo, al despedazar alguno de los subconjuntos con la medición de alguno de los síntomas. En la figura 4 las flechas correspondientes a los síntomas parten del subconjunto que despedazan Todas las rutas convergen en el nodo final, en el cual todos los subconjuntos han sido despedazados y cada subconjunto tiene un solo padecimiento. En la práctica no hay que generar toda la red, lo que se va generando son los nodos más promisorios utilizando algoritmos heurísticos desarrollados por investigadores de inteligencia artificial (Nilsson, 1971). Las distancias que se les asignan a las flechas son el producto del costo del síntoma por la suma de las probabilidades de los padecimientos que despedazan. Aplicándole a la red un algoritmo de rutas más cortas se determina la ruta óptima, la cual corresponde al 
árbol más económico. Para ilustrar, si suponemos que los costos y probabilidades son tales, que la ruta más corta resulta ser la que va más a la izquierda por las aristas en la figura 4, en la cual el primer síntoma que se mide es $S$, y luego para despedazar el subconjunto $\left(P_{1} P_{2}\right)$ se mide el síntoma $S_{3}$ y finalmente para despedazar el subconjunto $\left(\mathrm{P}_{3} \mathrm{P}_{4}\right)$ se mide el síntoma $\mathrm{S}_{2}$, entonces el árbol correspondiente es el de la figura 1. De esta manera se puede diseñar el árbol más económico.

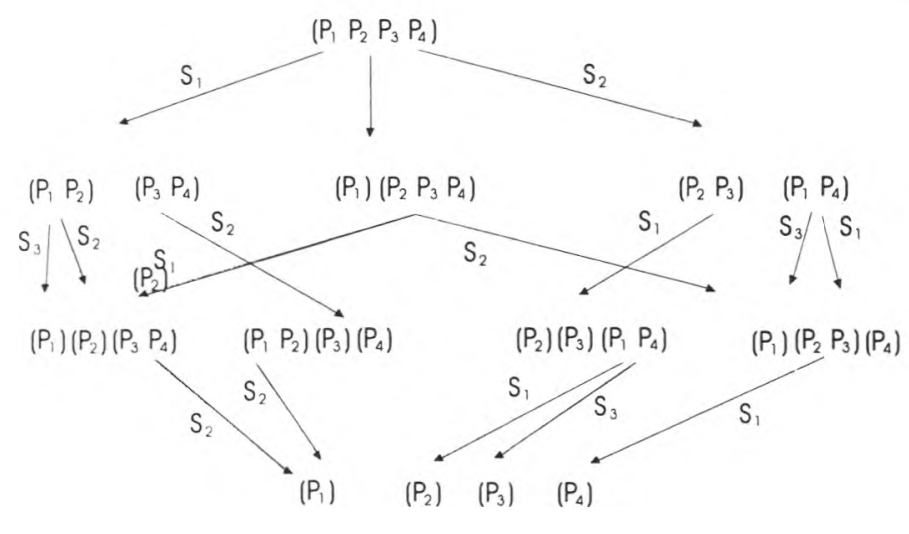

Figura 4

\section{Otra lección del acertijo de las monedas: diseño de códigos óptimos para comunicación}

En el área de codificación de señales digitales se presenta un problema muy similar al del diagnóstico médico con algunas simplificaciones. Se trata de darles códigos a los elementos de un alfabeto con el cual se construyen los mensajes, que ya codificados se envían por un sistema de transmisión de comunicación. El caso más simple de explicar es la codificación con secuencias binarias de las letras del alfabeto latino. Un análisis de textos en una lengua contemporánea nos indica que ciertas letras tienen una mayor frecuencia de aparición en un texto que otras. Por eiemplo, es sabido que en la lengua inglesa la letra con mayor frecuencia de aparición es la "e". Si le van a asignar a las letras códigos binarios con longitud variable es evidente que a las letras de mayor frecuencia se les deben dar códigos más cortos si se quiere minimizar la longitud de los mensajes transmitidos. Para que no haya necesidad de utilizar un código especial para separar las letras, lo cual aumentaría considerablemente el costo de la codificación, los códigos variables deben tener ciertas características, en cuyos detalles no tenemos espacio para adentrarnos (Hamming, 1997). Con estas características un mensaje se puede decodificar (sin costo adicional) instantáneamente. Para que el lector entienda la importancia de lo anterior, debe considerar un caso simple con 4 símbolos que se van a codificar en binario como sigue:

$$
\mathrm{s}_{1}=0, \mathrm{~s}_{2}=00, \mathrm{~s}_{3}=01, \mathrm{~s}_{4}=11
$$

Supóngase que se recibe el mensaje 0011. Dicho mensaje se podría interpretar como $s_{1} s_{1} s_{4}$ y también como $\mathrm{S}_{2} \mathrm{~S}_{4}$. Con esos códigos no se puede decidir. Supongamos ahora los códigos

$$
s_{1}=0, s_{2}=01, s_{3}=011, s_{4}=111
$$

Si se recibe el mensaje 0111111 . Este se puede decodificar sin ambigüedad y corresponde a $\mathrm{S}_{1} \mathrm{~S}_{4} \mathrm{~S}_{4}$, sin embargo, si le agregamos un 1 al final entonces el mensaje se convierte en $\mathrm{s}_{2} \mathrm{~s}_{4} \mathrm{~s}_{4}$. Nos percatamos de que hay que esperar la llegada del mensaje completo antes de poder decodificarlo, no obstante que no hay ambigüedad en los códigos. El código es no-ambiguo pero no decodificable instantáneamente. El siguiente código es no-ambiguo y decodificable instantáneamente:

$$
s_{1}=0, s_{2}=10, s_{3}=110, s_{4}=111
$$

y la decodificación se puede hacer en el momento que van llegando los bits. Por ejemplo, el mensaje

\section{0}

se decodifica como sigue:

$$
\begin{gathered}
110,10,0,10,0,110,111,0,0,0,10,10,0,110= \\
s_{3} S_{2} S_{1} S_{2} S_{1} S_{3} S_{4} S_{1} S_{1} S_{1} S_{2} S_{2} S_{1} S_{3}
\end{gathered}
$$

El código se puede representar por medio de un árbol decodificador muy parecido a los árboles de diagnóstico, como se muestra en la figura 5. 


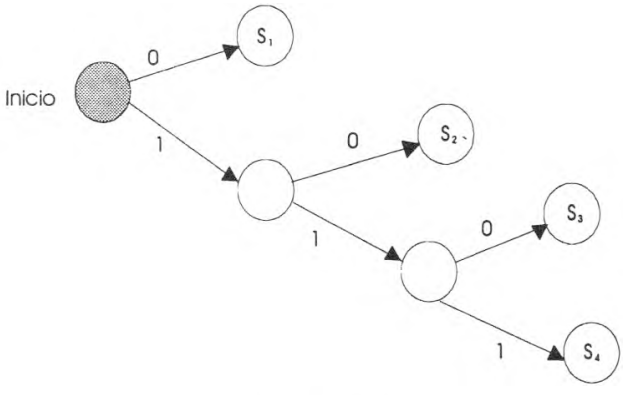

Figura 5.

Para decodificar con el árbol de la figura 5, se va leyendo el mensaje en binario iniciando en el círculo gris marcado con Inicio, tomando la rama correspondiente al dígito binario que vaya apareciendo. Para el mensaje utilizado como ejemplo, en el cual aparecen 1, 1, y 0 en los tres primeros lugares, las ramas que se toman son las primeras, dos inferiores y luego una inclinada hacia arriba. En ese momento, salimos del árbol con un símbolo $\left(\mathrm{S}_{3}\right)$, al cual registramos y continuamos donde nos habíamos quedado, Nótese la similitud con la operación de un árbol diagnóstico que es lo que nos permite utilizar las ideas aprendidas con el problema de las monedas para estudiar problemas de codificación. Dependiendo del código que se use será el árbol decodificador. Si usamos el siguiente código

$$
s_{4}=0, s_{3}=10, s_{2}=110, s_{1}=111
$$

aunque la estructura es muy similar, los símbolos quedan en diferentes partes del árbol y dependiendo de la frecuencia relativa (o probabilidad que se presenten en un mensaje) de cada símbolo, el costo promedio del árbol será diferente. Supóngase que los símbolos tienen las siguientes probabilidades de aparecer: $\mathrm{p}_{1}=0.5, \mathrm{p}_{2}=0.3, \mathrm{p}_{3}=0.15$, $\mathrm{p}_{4}=0.1$. Entonces el costo promedio del árbol de la figura 4 es

$$
\begin{gathered}
1(0.5)+2(0.3)+3(0.15)+4(0.1)=0.5+0.6+ \\
0.45+0.4=1.95
\end{gathered}
$$

El costo promedio del último código dado (al cual no le dibujamos árbol) sería:

$1(0.1)+2(0.15)+3(0.3)+4(0.5)=0.1+0.3+$ $0.9+2.0=3.3$
Vemos que hay una diferencia considerable en los costos, siendo el primer código el más económico. No siempre es conveniente que los árboles tengan la forma de la figura 4 en que en cada etapa se va decodificando un símbolo. Existe un algoritmo descubierto por Huffman (1952) que nos dice cómo diseñar códigos de mínimo costo, dadas las probabilidades de los símbolos. La explicación del algoritmo se dará por medio de un ejemplo sencillo. Supóngase que se quieren codificar cinco símbolos $\mathrm{s}_{1}, \mathrm{~s}_{2}, \mathrm{~s}_{3}, \mathrm{~s}_{4}, \mathrm{~s}_{5}$ cuyas probabilidades son: $\mathrm{p}_{1}=0.4, \mathrm{p}_{2}$ $=0.2, p_{3}=0.2, p_{4}=0.1, p_{5}=0.1$. Las probabilidades se colocan en una columna en orden descendente (columna más a la izquierda).

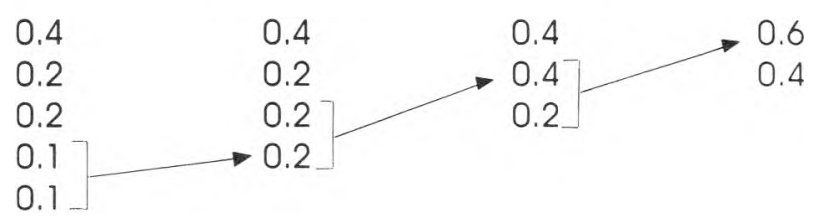

Luego se van creando columnas a la derecha aplicando la siguiente técnica:

Se encuentran las dos cantidades más pequeñas y se suman, creando una pareja cuya probabilidad es la suma. Dicha cantidad se coloca donde le corresponde de acuerdo con su tamaño y se escribe la nueva columna a la derecha. El procedimiento se repite con la nueva columna hasta que queden dos números en la misma. De las combinaciones que se hicieron se deduce el árbol decodificador notando cómo se fueron formando las diferentes cantidades. A las dos cantidades de la última columna se les asignan los códigos más cortos. Por ejemplo, al 0.4 le asignamos el código 1 y como el 0.4 corresponde totalmente al símbolo s, dicho símbolo queda con su código asignado. Como el 0.6 está formado con la combinación del 0.4 y 0.2 de la previa columna, al 0.6 le corresponde la cabeza de un árbol, que comienza con 0 y no un símbolo. El 0.2 proviene directamente del símbolo $s_{2}$ por lo tanto, a dicho símbolo le corresponde un código con 2 dígitos binarios (por ejemplo.01) el 0.4 será cabeza de un árbol que continúa. Dicho 0.4 proviene de dos componentes, un 0.2 que corresponde al símbolo $s_{3}$ al cual le corresponde un código con 3 dígitos binarios (por ejemplo 000) y el otro 0.2 corresponderá a otra cabeza de árbol, ya 
que proviene de los símbolos $\mathrm{s}_{4} \mathrm{y}_{5}$. A los dos les corresponderán códigos de cuatro dígitos binarios (0010 y 0011 ). El árbol decodificador se muestra en la figura 6.

El procedimiento de Huffman no produce necesariamente un árbol decodificador único, pues varias de las decisiones son discrecionales. Por ejemplo, cuando quedan empatadas las probabilidades de las combinaciones con las ya existentes, se pueden poner en diferentes posiciones, dando origen a árboles diferentes. Un segundo árbol decodificador de los mismos símbolos con iguales probabilidades se muestra en la figura 7 .

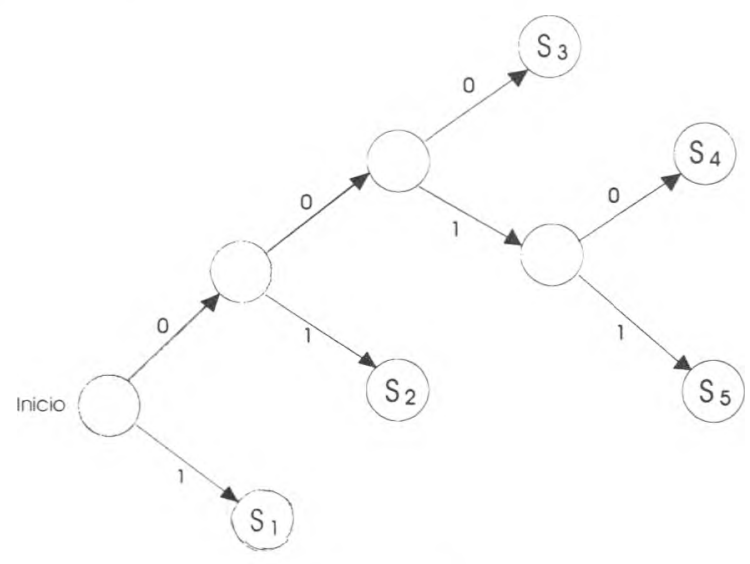

Figura 6

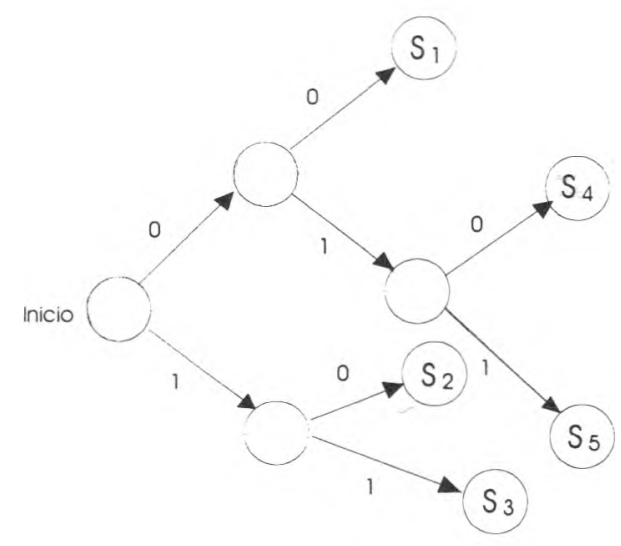

Figura 7

Aunque los árboles pueden ser distintos, el costo promedio de los arboles obtenidos con el algoritmo de Huffman son todos iguales. Así, el árbol de la figura 6 tiene un costo promedio de $1(0.4)+2(0.2)+3(0.2)+$ $4(0.1+0.1)=0.4+0.4+0.6+0.8=2.2$. El árbol de la figura 7 tiene un costo promedio de $2(0.4)+2(0.2$
$+0.2)+3(0.1+0.1)=0.8+0.8+0.6=2.2$. Como se ve, los costos promedio son iguales, aunque los códigos asignados a los símbolos sean diferentes. Para la figura 6 los códigos son: $s_{1}=1, s_{2}=01, s_{3}=$ $000, s_{4}=0010, s_{5}=0011$. Para la figura 7 los códigos son: $s_{1}=00, s_{2}=10, s_{3}=11, s_{4}=010, s_{5}=011$.

Como último punto observamos que independientemente de la búsqueda de códigos óptimos o no, no es difícil dar con códigos binarios no-ambiguos instantáneos, pues basta que se utilice un árbol para encontrarlo (Fano, 1961). Las puntas del árbol (llamadas hojas) nos dan los códigos. Por ejemplo, si deseamos codificar 6 símbolos podríamos utilizar alguno de los dos árboles que se muestran en la figura 8 .

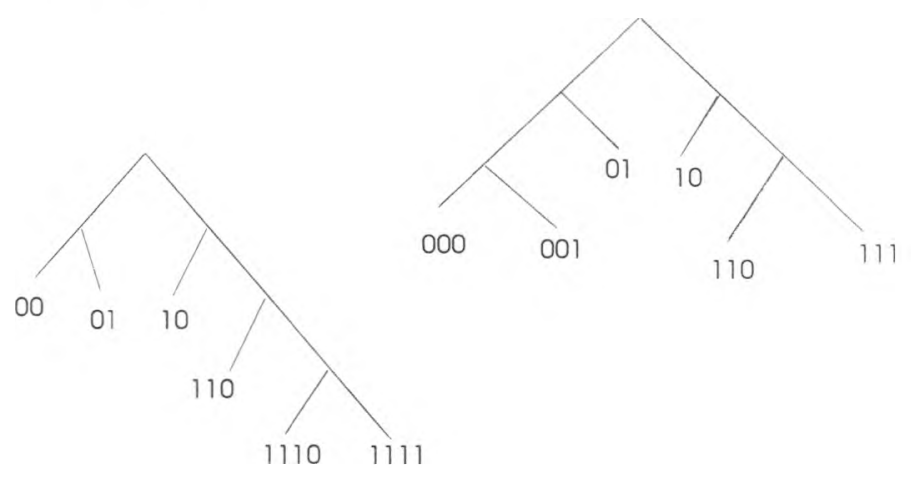

Figura 8

Con 6 símbolos existen 5 árboles diferentes; con 5 símbolos existen 3 árboles diferentes. La cuestión de cuántos árboles binarios diferentes existen para diferentes números de hojas se convierte en un problema matemático interesante para los especialistas de teoría de información. (Hamming, 1997); (Abramson, 1963); (Raisbeck, 1969).

\section{Conclusiones}

En cuanto a la aplicabilidad del acertijo de las doce monedas a diversas aplicaciones, hacemos notar que el acertijo corresponde a la familia de problemas de diagnóstico de sistemas, de la cual el diagnóstico médico es un caso particular. El diagnóstico de sistemas es importante y algunos autores han escrito libros completos al respecto (Court-Hare, 1967). Uno de los modelos de diagnóstico médico con el cual ilustramos lo que nos 
puede enseñar el acertijo de las monedas considera un conjunto de síntomas y hay que determinar la estrategia para hacer un diagnóstico bajo ciertas condiciones. En el acertijo de las monedas, los síntomas son los estados de la balanza después de cada experimento.

Existen otros modelos para manejar el diagnóstico médico, entre ellos, los conjuntos de reglas utilizados en los expertos artificiales y la aplicación de la regla de Bayes (Murray-Lasso, 1974). El modelo de diagnóstico en medicina se hizo más realista introduciendo probabilidades, las cuales se pueden estimar en una población determinada por medio de las frecuencias con las que se presentan ciertos padecimientos. Para el diagnóstico diferencial de algunos padecimientos se les podría asignar a la medición de los diferentes síntomas distintos costos (que pueden ser medidos en metálico o preferiblemente en unidades que también consideren aspectos como la molestia al paciente, el dolor, la dificultad de llevarlo a cabo, etc.). También para estudiar este punto hay investigación por parte de los especialistas en sistemas (Raiffa, 1968); (Churchman y Ackoff, 1954). Se puede entonces hablar de diagnóstico probable a costo mínimo, en el cual heurísticamente se hacen las mediciones de los síntomas que primero cuestan poco y solamente al final aquellos que cuestan más. Las ideas básicas, como llevar cuenta de las posibilidades que quedan por determinar y el número de puntas de los árboles, se pueden aplicar al problema del diagnóstico médico. También el acertijo de las monedas es factible al presentarse con conceptos probabilísticos y costos. Por ejemplo, en vez de requerir que la determinación de la moneda falsa se haga en tres pesadas, se pueden dar probabilidades diferentes para cada moneda de que sea falsa y hasta diferentes probabilidades de que sea ligera o pesada, así como costos diferentes para los distintos experimentos, además, pedir que se encuentre una estrategia de pesadas que minimice el costo esperado de encontrar la moneda falsa. De esta manera, si la moneda 1 tiene una altísima probabilidad de ser la falsa y el costo de un experimento es proporcional al número de monedas que se ponen en la balanza, puede resultar más conveniente pesar la moneda 1 contra la 2 y luego la
1 contra la 3 en los primeros dos experimentos, con una alta probabilidad de que la balanza se desequilibre en ambos, que dividir las monedas en grupos de 4 monedas cada uno como se hizo con el planteamiento original.

Hemos insistido en el ejemplo del diagnóstico médico por ser familiar a muchas personas, aunque no sean profesionales de la medicina. Sin embargo, el problema del diagnóstico se presenta en muchos sistemas en la práctica. Para determinar por qué no funciona un automóvil, el mecánico mide una serie de síntomas como por ejemplo, si le llega gasolina al carburador o si llega la chispa a las bujías. En caso de que no llegue gasolina al carburador, éste revisa si hay gasolina a la salida de la bomba de la misma. Si no llega chispa a las bujías, revisa si le llega electricidad a la bobina, etc. Un buen mecánico tiene en la cabeza un árbol diagnóstico parecido a los que aparecen en este artículo. Lo ha ido construyendo con la práctica y toma en consideración la frecuencia con la que se presentan ciertos problemas como mangueras con fugas, falsos contactos, platinos gastados, etc.

En forma similar procede un electricista al buscar una falla en un sistema eléctrico, si un foco no prende comienza por determinar si hay voltaje en los bornes o si otros focos de la casa prenden, pues si hay voltaje lo más probable es un foco fundido y si los otros focos no prenden, lo más probable es o un fusible fundido en el interruptor general o un apagón. Ciertamente no comienza sacando cables de los tubos conduit para determinar si algún alambre está cortado, pues la medición en esta forma, de este síntoma es muy costosa, existiendo síntomas casi equivalentes más baratos para llegar a las mismas conclusiones, por ejemplo, comprobando continuidad con un medidor de resistencias o una chicharra, haciendo conexiones con tierra u otros alambres. Solamente hasta haber encontrado falta de continuidad entre dos cajas de registro cercanas vale la pena considerar sacar o meter alambres en un tubo conduit.

Los ejemplos que se pueden dar son muy numerosos y van desde encontrar un bicho en un programa de computadora, una falla electrónica en una computadora, una falla en un sistema de comunicaciones o en un cohete aerospacial. 
DOI: http://dx.doi.org/10.22201/fi.25940732e.2002.03n3.014

El acertijo presentado en este artículo también tiene conexión con la teoría de la información. Cada pesada proporciona una cierta cantidad de información en la medida que elimina ciertas posibilidades y, si se consideran probables, aumentan las probabilidades de que resulten ser ciertas. Así, aunque en el problema de las 12 monedas el pesar 6 contra 6 en el primer experimento ofrece cierta cantidad de información como se señala en el artículo, el pesar cuatro contra cuatro da más información porque deja menos incertidumbre. En el problema de las 13 monedas se puede intuir que se requiere no desperdiciar nada de información por estar llenas las 27 puntas del árbol diagnóstico. En el de 12 monedas hay un poco de holgura pues todavía se puede meter una moneda más y determinar la falsa en tres pesadas.

Una segunda ilustración de aplicabilidad de las ideas del acertijo de las monedas se dio en el campo de la codificación de señales digitales. Se habló de una solución dada por Huffman que resuelve el problema con un sencillo algoritmo tan simple que se puede aplicar manualmente. Dado que el árbol como estructura, ya sea binario (con bifurcaciones de 2 ramas), o t-ario (ramificaciones con $t$ ramas), es una herramienta muy utilizada en computación, administración, sociología y otras disciplinas, existen muchas otras aplicaciones de lo aquí tratado. Por ejemplo, en el diseño de bases de datos en las cuales se conoce la frecuencia con la que se requiere encontrar las llaves de los registros, se diseñan los árboles de búsqueda utilizando el algoritmo de Huffman. Igual cosa sucede en el diseño de procesos de ordenamiento (sort) externo por medio de intercalado (merge) donde el algoritmo de Huffman proporciona soluciones óptimas (Knuth, 1968 y 1973).

Vemos, pues, que el paradigma que da el acertijo de la moneda falsa es rico en posibilidades y aplicaciones, lo que justifica su estudio detallado. Existen muchos paradigmas que vale la pena analizar. Un ejemplo en ingeniería es el sistema vibratorio de segundo orden (masa, resorte, amortiguador) en el que entran ideas básicas de amplia aplicabilidad en sistemas mecánicos, eléctricos, neumáticos e hidráulicos, como frecuencia natural, resonancia, análisis en el dominio de la frecuencia, amortiguamiento, constante de tiempo, transformación y almacenamiento de la energía de un tipo en otro, así como disipación de energía, por mencionar algunas (Cannon, 1967). La tesis de este autor es que el arte de resolver problemas consiste en conocer a profundidad la solución de suficientes paradigmas para cubrir un área dada del conocimiento. En este artículo se manejó una introducción a uno de estos paradigmas. La mayor parte de lo aprendido tiene que ver con matemáticas, aunque no necesariamente con las que tradicionalmente se le han impartido a los estudiantes de ingeniería. Conviene que los estudiantes dedicados a esta materia se percaten que hay mucho en las matemáticas que no es tan formal como el cálculo, el álgebra o la probabilidad (Davis y Hersh, 1981).

La computadora proporciona la oportunidad de medir experimentalmente la profundidad de comprensión de un paradigma, de ahí una buena parte de su valor didáctico. El convertirse en un buen profesional no es solamente cuestión de conocimientos. Una buena parte es cuestión de actitudes. El resolver un problema complejo como el de las 12 monedas requiere adquirir una actitud importante en ingeniería: paciencia y tenacidad. Un buen técnico o ingeniero tiene la paciencia y tenacidad de interesarse en un problema hasta que logra resolverlo. Muchas personas se dan por vencidas si no logran una solución satisfactoria en poco tiempo. La programación de computadoras y la prueba de los programas pueden promover las virtudes de la paciencia y tenacidad mencionadas Al que ha intentado escribir un programa complejo y logra su funcionamiento correcto después de un esfuerzo considerable, el ejercicio le proporciona una mayor auto estima. Dicha persona siente que es capaz de resolver problemas. Este individuo no se dará por vencido con facilidad cuando se le presente otro reto similar o mayor. La diferencia de desarrollo entre unos países y otros se debe, por lo menos en parte, a una desigualdad en paciencia y tenacidad en el proceso de solución de los problemas que se les presentan.

\section{Referencias}

Allman W.F. (1989). Apprentices of Wonder: Inside the Neural Network Revolution. Bantam Books, New York. 
Abramson N. (1963). Information Theory and Coding. Mc Graw-Hill Book Company, New York.

Court-Hare V. Ir. (1967). Systems Analysis: A Diagnostic Approach. Harcout, Brace and World, Inc., New York. Churchman C.W. y Ackoff R.L. (1954 ). An Approximate Measure of Value. Operations Research, Vol. 2, 172-181. Cannon R.H. Jr. (1967). Dynamics of Physical Systems. Mc Graw-Hill Book Company, New York.

Davis P.J. y Hersh R. (1981). The Mathematical Experience. Houghton Mifflin Company, Boston, MA.

De Vecchi B. (1972). Otra solución al problema de las 12 bolas y la aplicación de su metodología al diagnóstico médico. Revista Mexicana de Electricidad, Vol. 33, No. 378, 84-88.

Dijkstra E.W. (1959). A Note on Two Problems in Connection with Graphs. Num. Math, Vol. 1, 269-271.

Fano R. (1961).Transmission of Information, John Wiley \& Sons, Inc., New York.

Floyd R.W. (1962). "Algorithm 97: Shortest Path" Comunications of the ACM., Vol. 5, 345.

Hammina R.W. (1997). The Art of Doing Science and Engineering, Gordon and Breach Science Publishers, Amsterdam.

Hopfield I.J. (1982). "Neural Neetworks and Physical Systems with Engineering Collective Computational Abilities" Proc. Nat. Acad. Sci., Vol. 79, 2554-58. Hu T.C. (1969). Integer Programming and Network Flows. Addison-Wesley Publishing Company, Reading, MA. Knuth D.E. (1968). The Art of Computer Programming, Vol. I, Fundamental Algorithms. Addison-Wesley Publishing Company, Reading, MA.

Knuth D.E (1973). The Art of Computer Programming, Vol. III, Sorting and Searching. Addison-Wesley Publishing Company, Reading, MA.
Lara-Tejeda J. (1977). Diseño de árboles óptimos de diagnóstico con técnicas de inteligencia artificial. Tesis de Maestría en Investigación de Operaciones, División de Estudios de Posgrado de la Facultad de Ingeniería, Universidad Nacional Autónoma de México.

Ledley R.S. y Lusted L.B. (1959). Reasoning Foundations of Medical Diagnosis. Science, Vol. 5, No. 130, 9-21.

Levine R.I., Drang D.E. y Edelson B. (1986). A Comprehensive Guide to AI and Expert Systems. Mc Graw-Hill Book Company, New York.

Murray-Lasso M.A. (1972). Aplicación de la metodología de árboles de decisión e información al diagnóstico médico. Revista Mexicana de Electricidad, Vol. 32, No. 383, 405-407, agosto; y No $384,447-451$ y 478-479, septiembre. Boletín del Instituto Mexicano de Planeación y Operación de Sistemas. Vol. 3, No. 14, 12-32, noviembre-diciembre 1972

Murray-Lasso M.A. (1974). Aplicación de la teoría de decisiones al diagnóstico en medicina. Boletín del Instituto Mexicano de Planeación y Operación de Sistemas, Vol. 4, No. 24, julio-agosto, 121-137.

Murray-Lasso M.A. y Lara J. (1975). Diagnóstico óptimo por computadora aplicado a la medicina. Memoria del I Congreso de la Academia Nacional de Ingeniería, Guanajuato, Gto., junio.

Nilsson N.J. (1971). Problem Solving Methods in Artificial Intelligence. Mc Graw-Hill Book Company, New York. Raiffa H. (1968). Decision Analysis. Addison-Wesley Publishing Company, Reading, MA.

Raisbeck G. (1969). Information Theory: An Introduction for Scientists and Engineers. MIT Press, Cambridge, MA.

Shortliffe E.H. (1976). Computer-Based Medical Consultations: MYCIN. American Elsevier, New York.

\section{Semblanza del autor}

Marco Antonio Murray-Lasso. Realizó la licenciatura en ingeniería mecánica-eléctrica en la Facultad de Ingeniería de la UNAM. El Instituto de Tecnología de Massachussetts (MIT) le otorgó los grados de maestro en ciencias en ingeniería eléctrica y doctor en ciencias cibernéticas. En México, ha laborado como investigador en el Instituto de Ingeniería y como profesor en la Facultad de Ingeniería (UNAM) durante 40 años; en el extranjero; ha sido asesor de la NASA en diseño de circuitos por computadora para aplicaciones espaciales, investigador en los Laboratorios Bell, así como profesor de la Universidad Case Western Reserve y Newark College of Engineering, en los Estados Unidos. Fue el presidente fundador de la Academia Nacional de Ingeniería de México; vicepresidente y presidente del Consejo de Academias de Ingeniería y Ciencias Tecnológicas (organización mundial con sede en Washington que agrupa las Academias Nacionales de Ingeniería) y secretario de la Academia Mexicana de Ciencias. Actualmente es jefe de la Unidad de Enseñanza Auxiliada por Computadora de la División de Estudios de Posgrado de la Facultad de Ingeniería de la UNAM, investigador nacional en ingeniería, consejero educativo del MIT y consultor de la UNESCO 


\section{Instructions for authors}

\section{Purpose and scope of the magazine}

The purpose of Ingeniería. Investigación y Tecnología magazine is to spread original and unpublished information and knowledge related to all fields of engineering. It provides a forum to professors, researchers, practitioners and students from all over the world to submit for publication articles in either Spanish or English.

\section{The contributions will be classified in either one of the three following sections:}

Engineering in Mexico and around the world. Reviews and discussions related to the development, evolution and progress made in engineering issues of technical, scientific, epistemological and professional nature

Recent studies and research. Papers written with a methodological, analytical, numerical and experimental approach as well as those characterized by technological innovation and development of practical application to education, professional activities and research work related to engineering.

Engineering and society. Analysis and discussion of social and professional issues related explicitly to engineering.

\section{Manuscripts}

1. The final original manuscripts properly written in Spanish or English should be submitted in a diskette with ASCII, Corel Ventura or Word format together with three hard legible copies with double-spaced printing and $3-\mathrm{cm}$ wide all-around margins. Illustrations should be submitted in ZIP encapsulated form indicating the format in which they were created.

2. The heading of the article should include the following information: title of the paper; full name of author(s); present position; E-mail address, postal address, telephone and fax numbers of the author who will receive the correspondence; a list of keywords and a summary in both Spanish and English. The summary of no more than 200 words, should provide a concise, exact and unambiguous idea of the contents and scope of the article.

3. Sections and subsections of the article should be clearly differentiated but not numbered. Introduction: The subject, scope and purpose of the work should be described in lay language. Main body of the text: It should contain the plan of development of the subject matter in the paper, organized in a logic and simple manner; subdivisions of the text should include a heading without numbering. Conclusions: They should state concisely the contributions of the paper, as well as its scope, limitations and applicability. Acknowledgements: To be brief and concise. References and appendices.

4. The maximum length of the article should not exceed eight printed pages of text (equivalent to about 6500 words), or 10 pages including tables and figures.

5. Figures, photographs and tables should be provided as originals attached to the manuscript although not pasted into the text, but rather in separate folders and sheets and with dimensions compatible with the page size of the publication. The illustrations should be referred to as figures to be progressively numbered as mentioned in the text and identified as Fig. 1, $2, \ldots$ or figure $1,2, \ldots$ Number tables in the same order in which they are mentioned in text and identify them as table 1,2 ,

6. All mathematical expressions must be clearly typed, and care should be taken to differentiate the symbols unambiguously. It shall be compulsory to use the SI system of units.

7. For the references in the main body of the text give the last name(s) of the author(s) and the year of publication. For example: "Recently, Gracia-Sánchez and Estrada-Serrano (1996)..." or "In a recent paper (Gracia-Sánchez and Estrada-Serrano, 1996)..." All references must appear together at the end of the paper listed alphabetically by last name of the first author according to the following general rules:

\section{Periodical publications}

Sotelo G. and Cafaggi A. (1996). Cálculo de la dimensión mínima de la sección de un canal o de los tirantes alternos, cuando se conocen el gasto y la energía específica. Ingeniería, Vol. LXVI, 25-46.

\section{Books}

Rosenblueth E. (1980). Sobre ciencia e ideología. Fundación Javier Barros Sierra, Mexico.

\section{Contributions to books}

Carrera-Bolaños J. (1994). Racionalidad, tecnología y desarrollo En Los grandes problemas de la ciencia y la tecnología (Varela R. and Mayer L. Ed.), pp.39-47. Universidad Autónoma Metropolitana, Mexico.

\section{Conference Reference}

Ayala G., Muñoz C. and Esteva L. (1984). Determination for Earthquake Design Parameters for Different Local and Topographic Conditions. $8^{\text {th }}$ World Conference on Earthquake Engineering, San Francisco, California, USA, July.

\section{Unpublished reports/Theses or dissertations}

Iturbe-Argüelles M. del R. (1997). Transporte y dispersión de compuestos químicos orgánicos (hidrocarburos asfálticos halogenados) a través del flujo en medio poroso. PhD thesis in Engineering. Universidad Nacional Autónoma de México, Ciudad Universitaria, Mexico.

\section{Web sites}

Anderson S. (1997). Multimedia on the Internet: A course designed to study the tools of multimedia on the Word Wide Web. http://www.usu.edu/ sanderso/multinet/

8. A brief résumé of no more than 100 words should be included for each of the authors.

\section{Arbitration}

All articles submitted to the magazine should be reviewed by three arbitrators who will evaluate them and give an opinion about their publication. The Task Committee is integrated by top specialists with wide recognition in the different areas of expertise and trained to provide a reliable and expeditious evaluation of the quality and originality as well as of the value and interest of the paper contents

\section{Proofreading of galleys}

Authors will receive the dummy of their articles for a final proofreading, together with a format of "assignment of copyright ownership and the right to reprint the articles".

\section{Reprints}

Ten copies of each article published will be sent on a complimentary basis to the senior author.

\section{Mailing of manuscripts}

The delivery of an article binds the author(s) to refrain for submitting it simultaneously for review at any other magazine or journal.

Manuscripts should be submitted for review by mailing them to the following address: Ingeniería. Investigación y Tecnología, Edificio Principal de la Facultad de Ingeniería, UNAM. Biblioteca Antonio Dovalí Jaime,Ciudad Universitaria,Coyoacán 04510 , México, DF. 